\title{
Copy-number variations in Y-chromosomal azoospermia factor regions identified by multiplex ligation-dependent probe amplification
}

\author{
Kazuki Saito ${ }^{1,2,3}$, Mami Miyado ${ }^{1}$, Yoshitomo Kobori ${ }^{4}$, Yoko Tanaka ${ }^{5}$, Hiromichi Ishikawa ${ }^{6}$, Atsumi Yoshida ${ }^{7}$, \\ Momori Katsumi ${ }^{1}$, Hidekazu Saito ${ }^{3}$, Toshiro Kubota ${ }^{2}$, Hiroshi Okada ${ }^{4}$, Tsutomu Ogata ${ }^{1,8}$ and Maki Fukami ${ }^{1}$
}

Although copy-number variations (CNVs) in Y-chromosomal azoospermia factor (AZF) regions have been associated with the risk of spermatogenic failure (SF), the precise frequency, genomic basis and clinical consequences of these CNVs remain unclear. Here we performed multiplex ligation-dependent probe amplification (MLPA) analysis of 56 Japanese SF patients and 65 control individuals. We compared the results of MLPA with those of conventional sequence-tagged site PCR analyses. Eleven simple and complex CNVs, including three hitherto unreported variations, were identified by MLPA. Seven of the 11 CNVs were undetectable by conventional analyses. CNVs were widely distributed in AZF regions and shared by $\sim 60 \%$ of the patients and $\sim 40 \%$ of the controls. Most breakpoints resided within locus-specific repeats. The majority of CNVs, including the most common gr/gr deletion, were identified in the patient and control groups at similar frequencies, whereas simple duplications were observed exclusively in the patient group. The results imply that AZF-linked CNVs are more frequent and heterogeneous than previously reported. Non-allelic homologous recombination likely underlies these CNVs. Our data confirm the functional neutrality of the gr/gr deletion in the Japanese population. We also found a possible association between AZF-linked simple duplications and SF, which needs to be evaluated in future studies.

Journal of Human Genetics (2015) 60, 127-131; doi:10.1038/jhg.2014.115; published online 8 January 2015

\section{INTRODUCTION}

Copy-number variations (CNVs) in azoospermia factor (AZF) a-c regions have been associated with the risk of spermatogenic failure (SF). ${ }^{1,2}$ Of these, submicroscopic deletions in AZFb and/or AZFc regions represent the major genetic causes of $\mathrm{SF}$, although they are also observed in a small proportion of normozoospermic individuals. ${ }^{3}$ $\mathrm{AZFb}$ and $\mathrm{AZFc}$ regions are enriched with several locus-specific repeats and are highly susceptible to non-allelic homologous recombination. ${ }^{3,4}$ Deletions in these regions are predicted to cause $\mathrm{SF}$ by reducing the copy-number of multi-copy genes such as $D A Z$, CDY1/2 and TSPY1. ${ }^{5-8}$ To date, deletions in AZFb/c regions were analyzed mostly by PCR of sequence-tagged site (STS) markers (STSPCR). ${ }^{9}$ In 2011, Rozen et al. ${ }^{10}$ performed STS-PCR for 20884 male individuals with and without SF and identified four types of AZFc deletions in $3.7 \%$ of the subjects. Rozen et al. ${ }^{10}$ revealed that most of these deletions exert negative effects on spermatogenesis; the most common deletion referred to as the gr/gr deletion almost doubled the risk of SF, whereas a deletion referred to as the b2/b4 deletion increased the risk by a factor of 145 . Other researchers have also confirmed the pathogenicity of $\mathrm{AZFb} / \mathrm{c}$ deletions. ${ }^{3,11}$ However, the frequencies and phenotypic effects of these deletions seem to differ among ethnic groups. ${ }^{11-13}$

Other types of CNVs in AZF regions, such as microduplications and complex deletion-duplication rearrangements, have also been reported. ${ }^{14-18}$ However, the number of these reports is limited, because of technical difficulties in identifying such CNVs. Indeed, conventional STS-PCR has focused on deletions in AZFb and AZFC regions. Thus, the precise frequency, genomic basis and clinical consequences of AZF-linked CNVs remain largely unknown. For example, Giachini et al. ${ }^{18}$ found no significant pathogenic effect of AZFc duplications, whereas Lin et al. ${ }^{16}$ associated these duplications with a significant risk of oligospermia.

Recent advances in molecular techniques, including the development of multiplex ligation-dependent probe amplification (MLPA) and comparative genomic hybridization, have enabled researchers to identify CNVs at multiple loci in a single assay..$^{19,20}$ In 2012, Bunyan et al. ${ }^{21}$ employed MLPA for the detection of CNVs in AZF regions. They studied 50 SF patients and 50 control individuals and identified

${ }^{1}$ Department of Molecular Endocrinology, National Research Institute for Child Health and Development, Tokyo, Japan; ${ }^{2}$ Department of Comprehensive Reproductive Medicine, Tokyo Medical and Dental University, Tokyo, Japan; ${ }^{3}$ Division of Reproductive Medicine, Center for Maternal-Fetal-Neonatal and Reproductive Medicine, National Medical Center for Children and Mothers, Tokyo, Japan; ${ }^{4}$ Department of Urology, Dokkyo Medical University Koshigaya Hospital, Koshigaya, Japan; ${ }^{5}$ Department of Pediatrics, Tokyo Dental College Ichikawa General Hospital, Ichikawa, Japan; ${ }^{6}$ Reproduction Center, Tokyo Dental College Ichikawa General Hospital, Ichikawa, Japan; ${ }^{7}$ Reproduction Center, Kiba Park Clinic, Tokyo, Japan and ${ }^{8}$ Department of Pediatrics, Hamamatsu University School of Medicine, Hamamatsu, Japan

Correspondence: Dr M Fukami, Department of Molecular Endocrinology, National Research Institute for Child Health and Development, 2-10-1 Ohkura, Setagayaku, Tokyo 157-8535, Japan. 


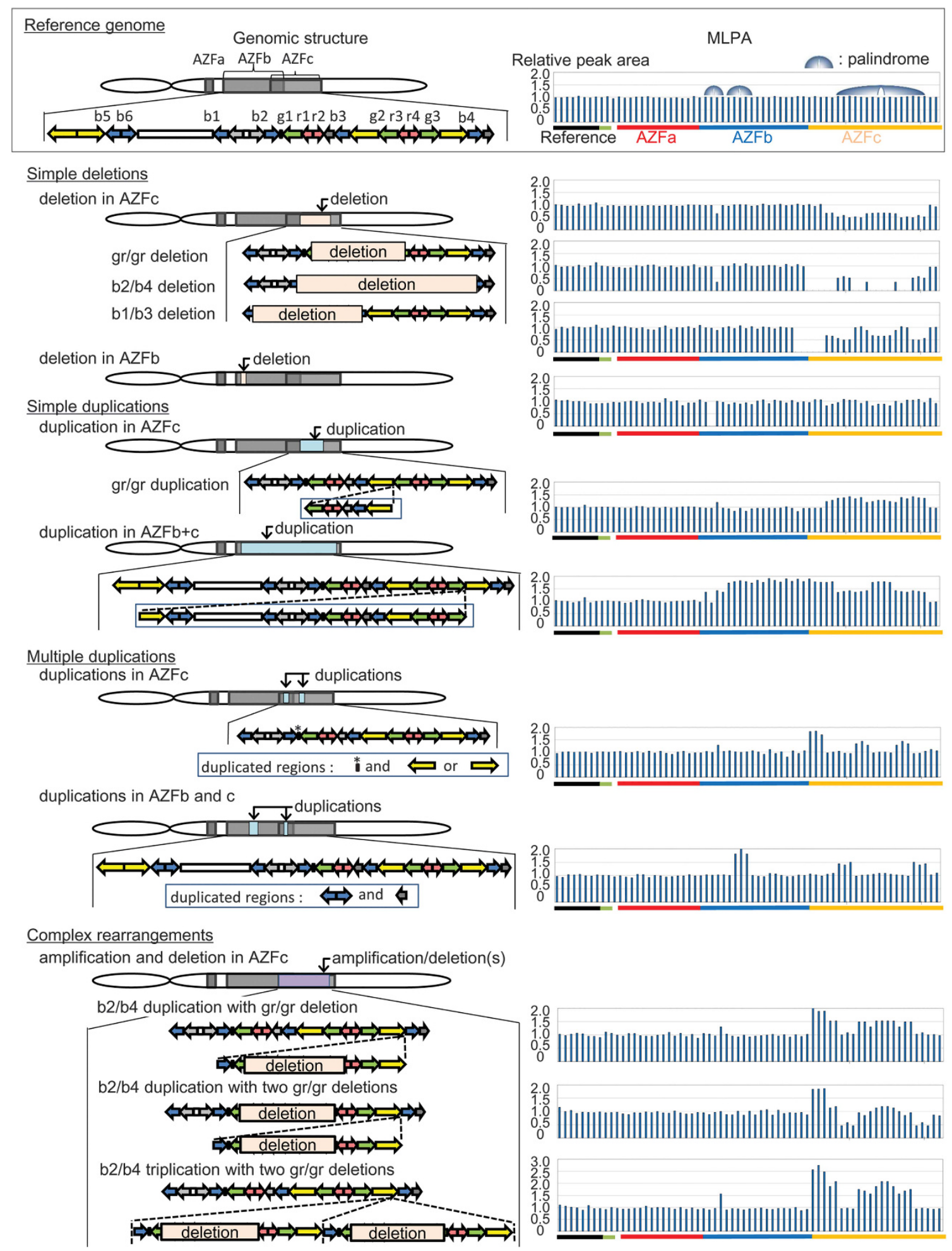

Figure 1 Copy-number variations in azoospermia factor (AZF) regions identified by multiplex ligation-dependent probe amplification (MLPA). Left panel: Schematic representation of the genomic structure of AZF regions. The colored arrows depict the locus-specific repeats in AZFb/c regions. The structure of the reference genome is based on the sequence retrieved from GenBank. Right panel: Representative results of MLPA. The relative peak areas were calculated by dividing each peak area of the samples by the average of that of five reference samples. Decreased and increased relative peak areas suggest copy-number loss and gain, respectively. 
four types of simple CNVs that were undetectable by STS-PCR. Likewise, Liu et al. ${ }^{22}$ performed MLPA analysis of samples obtained from 199 fathers and their sons. Although Liu et al. identified AZFlinked deletions in $7.5 \%$ of the fathers, they did not analyze the presence or absence of copy-number gains. There have been no further reports of MLPA analysis for AZF regions. Here we preformed MLPA on 121 Japanese individuals with and without SF.

\section{SUBJECTS AND METHODS}

\section{Subjects}

A total of 121 unrelated Japanese individuals participated in the present study. The patient group consisted of 56 men who were diagnosed with idiopathic non-obstructive azoospermia or oligospermia. All patients visited our clinics because of infertility. Patients with cytogenetically detectable chromosomal abnormalities were excluded from this study. The control group included 65 men who have fathered at least one child.

\section{Molecular analysis}

This study was approved by the Institutional Review Board Committee at the National Center for Child Health and Development and performed after taking written informed consent from the participants. Genomic DNA samples were obtained from peripheral leukocytes. MLPA was performed using SALSA MLPA probe-mix kit P360-A1 (MRC-Holland, Amsterdam, the Netherlands), according to the manufacturer's instructions. This kit contained 43 specific probes for AZF regions, together with 8 reference probes for other genomic regions (Supplementary Figure 1). The MLPA products were analyzed using a GenomeLab GeXP gene analysis system (Beckman Coulter, Fullerton, CA, USA). The relative peak area of each probe was calculated by dividing the actual peak area of the subject by the average of that of five reference samples. The results were confirmed by a second experiment.

To evaluate the usefulness of MLPA in the detection of AZF-linked CNVs, we compared the results of MLPA with those of conventional STS-PCR. In this study, five STS markers in AZFc region, sY1191, sY1291 sY1192, sY1189 and sY254, were analyzed as described previously. ${ }^{10}$

\section{Statistical analysis}

Statistical differences in the frequencies of CNVs between the patient and control groups were analyzed using $\chi^{2}$ and Fisher's exact tests. Differences in the frequencies of copy-number gain of multi-copy genes were also analyzed using Fisher's exact tests. $P$-values $<0.05$ were considered significant.

\section{RESULTS}

Eleven types of CNVs were identified in 58 of the 121 individuals (Figure 1; Table 1). The $11 \mathrm{CNVs}$ consisted of four simple deletions, two simple duplications, two multiple duplications (two nonoverlapping duplications on one allele) and three complex rearrangements. The three complex rearrangements were assumed to be duplication or triplication of the genomic region between b2 and b4, which harbored the gr/gr deletion (Supplementary Figure 2). Most of the $11 \mathrm{CNV}$ s involved genomic intervals in AZFc region, whereas two CNVs affected both AZFb and AZFc regions and one deletion involved only a small genomic interval in $\mathrm{AZFb}$ region. Three of the $11 \mathrm{CNVs}$, that is, a simple deletion in $\mathrm{AZFb}$ region, a simple duplication in $\mathrm{AZFb}+\mathrm{c}$ region and multiple duplications in $\mathrm{AZFb}$ and $A Z F c$ regions, have not been reported previously. The breakpoints of $10 \mathrm{CNV}$ s were located within AZF-specific repeats, whereas those of multiple duplications in AZFc region remained to be determined (Table 1).

Of the 11 CNVs detected by MLPA, only four were identified by STS-PCR (Figure 2). Three simple deletions in AZFc region (the gr/gr, b2/b4 and b1/b3 deletions) involving one or more of the five STS markers were detected by STS-PCR. One of the complex rearrangements (the b2/b4 duplication combined with gr/gr deletions) was assessed as a simple gr/gr deletion. Other CNVs, that is, a deletion in $\mathrm{AZFb}$ region, two of the three complex rearrangements and all duplications, yielded apparently normal results in STS-PCR.

Table 1 Copy-number variations identified in the present study

\begin{tabular}{|c|c|c|c|c|c|c|c|c|c|c|}
\hline & \multirow{2}{*}{$\begin{array}{c}\text { Patient, } \\
\mathrm{n}=56\end{array}$} & \multirow{2}{*}{$\begin{array}{l}\text { Control, } \\
n=65\end{array}$} & \multirow{2}{*}{$\begin{array}{c}\text { Statistical } \\
\text { significance }\end{array}$} & \multicolumn{2}{|c|}{ Predicted position of the breakpoints } & \multicolumn{5}{|c|}{ Estimated gene copy-number ${ }^{\mathrm{a}}$} \\
\hline & & & & Proximal & Distal & $D A Z(4)$ & CDY1 (2) & CDY2 (2) & HSFY (2) & USP9Y (1) \\
\hline Any copy-number alteration & 33 & 25 & $P=0.025$ & & & & & & & \\
\hline Simple deletion & 25 & 22 & $P=0.224$ & & & & & & & \\
\hline gr/gr deletion & 23 & 20 & & gr-repeat $^{\mathrm{b}}$ & gr-repeat $^{\mathrm{b}}$ & 2 & 1 & 1 & 2 & 1 \\
\hline b2/b4 deletion & 1 & 1 & & b2-repeat & b4-repeat & 0 & 0 & 2 & 2 & 1 \\
\hline b1/b3 deletion & 0 & 1 & & b1-repeat & b3-repeat & 2 & 2 & 2 & 2 & 1 \\
\hline deletion in AZFb & 1 & 0 & & y4-repeat & y4-repeat & 4 & 2 & 1 or 2 & 2 & 1 \\
\hline Simple duplication & 4 & 0 & $P=0.043$ & & & & & & & \\
\hline gr/gr duplication & 3 & 0 & & gr-repeat $^{\mathrm{b}}$ & gr-repeat $^{b}$ & 6 & 3 & 2 & 2 & 1 \\
\hline duplication involving $\mathrm{AZFb}+\mathrm{C}$ & 1 & 0 & & y3-repeat & y2-repeat & $8 ?$ & 3 & 3 & 4 & 1 \\
\hline Multiple duplications & 1 & 1 & $P=0.713$ & & & & & & & \\
\hline duplications in AZFc & 1 & 0 & & Unknown & Unknown & 4 & 3 & 2 & 2 & 1 \\
\hline duplications in AZFb and C & 0 & 1 & & $\begin{array}{c}\text { Repeat } \\
\text { sequence }^{c}\end{array}$ & $\begin{array}{c}\text { Repeat } \\
\text { sequence }^{c}\end{array}$ & 4 & 2 & 2 & 2 or 4 & 1 \\
\hline Complex rearrangements & 3 & 2 & $P=0.331$ & & & & & & & \\
\hline b2/b4 triplication with gr/gr deletions & 1 & 0 & & $\begin{array}{c}\text { Repeat } \\
\text { sequence }^{c}\end{array}$ & $\begin{array}{c}\text { Repeat } \\
\text { sequence }^{c}\end{array}$ & 8 & 4 & 2 & 2 & 1 \\
\hline b2/b4 duplication with gr/gr deletions & 1 & 0 & & $\begin{array}{c}\text { Repeat } \\
\text { sequence }^{c}\end{array}$ & $\begin{array}{c}\text { Repeat } \\
\text { sequence }^{c}\end{array}$ & 4 & 2 & 2 & 2 & 1 \\
\hline b2/b4 duplication with gr/gr deletion & 1 & 2 & & $\begin{array}{c}\text { Repeat } \\
\text { sequence }^{c}\end{array}$ & $\begin{array}{c}\text { Repeat } \\
\text { sequence }^{c}\end{array}$ & 6 & 3 & 2 & 2 & 1 \\
\hline
\end{tabular}

aThe numbers in the parentheses indicate copy-number of the genes in the human reference sequence.

bThe breakpoints reside within g- or r-repeat sequences.

${ }^{\mathrm{c}}$ All of the multiple breakpoints reside within repeat sequences. 


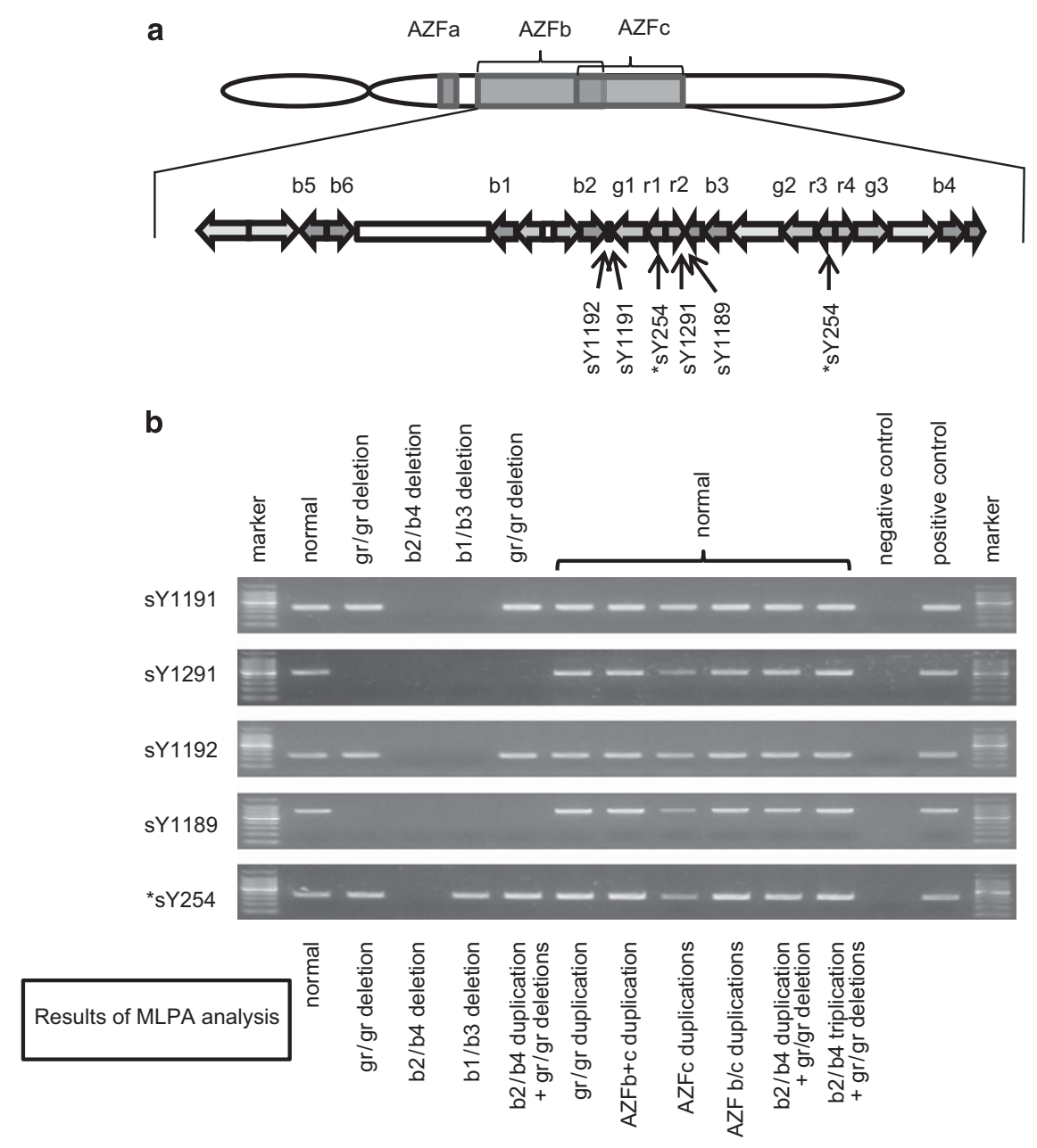

Figure 2 PCR analyses for sequence-tagged site (STS) markers. (a) Genomic positions of five STS markers. Of the five markers, sY254 is located at multiple sites (asterisks). (b) Representative results of STS-PCR. This method was able to identify four of the 11 copy-number variations identified by multiplex ligation-dependent probe amplification (MLPA). AZF, azoospermia factor. A full color version of this figure is available at the Journal of Human Genetics journal online.

CNVs were more frequently identified in the patient group (33 of $56,58.9 \%$ ) than in the control group ( 25 of $65,38.5 \% ; P=0.025$; Table 1). Although simple deletions and complex rearrangements were observed in the two groups at similar frequencies $(P=0.224$ and $P=0.331$, respectively), simple duplications were detected exclusively in the patient group $(P=0.043)$. Multiple duplications were identified in one patient and one control male. The frequencies of copy-number gain of multi-copy genes were similar between the two groups (Supplementary Table 1).

\section{DISCUSSION}

We performed MLPA for 121 Japanese individuals and identified 11 types of CNVs in AZF regions. These CNVs were detected in $\sim 60 \%$ of SF patients and in $\sim 40 \%$ of control individuals. The total frequency of CNVs was higher than that reported in previous studies on the Japanese population. ${ }^{11,23}$ Notably, 7 of the $11 \mathrm{CNV}$ s identified by MLPA were undetectable by STS-PCR. In addition, MLPA was capable of characterizing a complex structure of the b2/b4 duplication-gr/gr deletions, which was assessed as a simple gr/gr deletion by STS-PCR. These results demonstrate the usefulness of MLPA in the identification and characterization of AZF-linked CNVs. As MLPA is a relatively simple method and requires only a small amount of genomic
DNA, ${ }^{19}$ it can be used for the molecular diagnosis of several clinical samples. MLPA analysis appears to be beneficial for patients with SF, because it has been suggested that detection of AZF-linked CNVs would help to predict the sperm recovery rate at testicular sperm extraction. ${ }^{9}$

The $11 \mathrm{CNV}$ s identified in this study were widely distributed in AZF regions and included three rearrangements that have not been reported previously. Our findings provide further evidence for a high frequency and genetic heterogeneity of AFZ-linked CNVs. Notably, 10 of the $11 \mathrm{CNVs}$ had their breakpoints within AZF-specific repeats, while the breakpoints of the multiple duplications in AZFc region remain to be determined. These data support the previously proposed notion that non-allelic homologous recombination has a critical role in the development of CNVs in AZF regions. ${ }^{24}$

Two matters are noteworthy for the frequencies of CNVs in the patient and control groups. First, the most common gr/gr deletion was detected in 23 of 56 patients (41.1\%) and in 20 of 65 controls (30.8\%). Previous studies have shown that the frequency of the gr/gr deletion in control subjects is variable among ethnic groups, ranging from $0.0 \%$ in the Dutch to $33.9 \%$ in the Japanese. ${ }^{18}$ It was suggested that the gr/gr deletion is accompanied by multiple haplogroups of diverse pathogenicity, and this deletion in Japanese individuals is usually 
accompanied by a functionally neutral haplogroup. ${ }^{11,12}$ Our findings confirm that, in Japan, the gr/gr deletion represents a common variation that has a negligible effect on spermatogenesis. Second, although most of the $11 \mathrm{CNV}$ s were identified in the patient and control groups at similar frequencies, simple duplications were detected exclusively in the patient group. These results are consistent with those of a previous study on the Taiwan Han Chinese population in which the risk of SF was associated with AZF duplications but not with deletions. ${ }^{16,17}$ Copy-number gain of multi-copy genes, such as $D A Z$ and $C D Y 1$, or conformational changes of $\mathrm{Y}$ chromosome may exert deleterious effects on spermatogenesis. ${ }^{16,17}$ However, copynumber gain of the multi-copy genes would not be sufficient to cause SF, because two of our control individuals also had an increased copy-number of these genes (Table 1). Indeed, we found no significant difference in the frequencies of copy-number gain of multi-copy genes between the patient and control groups (Supplementary Table 1). Furthermore, USP9Y, a putative causative gene for $\mathrm{SF}^{25}$ was not affected in our patients.

Considering the small number of subjects in this study, the pathogenicity of AZF-linked CNVs, except for the gr/gr deletion, remains unclear. Furthermore, DNA samples of patients' relatives were not analyzed in this study. As previous studies have shown that de novo occurrence of $\mathrm{CNVs}$ in $\mathrm{AZF}$ regions is a relatively rare event, ${ }^{1,22,26}$ molecular analysis of the fathers of SF patients with CNVs would provide critical information regarding the effect of the CNVs on spermatogenesis. Further studies, including MLPA analysis in large cohorts and familial analyses of CNV-positive individuals, will clarify the clinical significance of each CNV.

In conclusion, the results expanded our understanding of the frequency and genetic heterogeneity of AZF-linked CNVs. It appears that non-allelic homologous recombination underlies most, if not all, $\mathrm{CNVs}$ in AZF regions. In addition, we confirmed the functional neutrality of the gr/gr deletion in Japanese individuals. We found a possible association between AZF microduplications and the risk of $\mathrm{SF}$, which needs to be evaluated in future studies.

\section{CONFLICT OF INTEREST}

The authors declare no conflict of interest.

\section{ACKNOWLEDGEMENTS}

We are grateful to the participants of this study. The study was supported by the Grants-in-Aid from the Ministry of Education, Culture, Sports, Science and Technology and from the Japan Society for the Promotion of Science and by the Grants from the Ministry of Health, Labor and Welfare (Grant Numbers: H26-062, 082, Tokyo, Japan) from National Center for Child Health and Development (Grant Numbers: 26-11, 26-19, Tokyo, Japan) and from Takeda foundation. The sponsors had no role in study design, in the collection, analysis or interpretation of data, in the writing of the report or in the decision to submit the article for publication.

1 Vogt, P. H., Edelmann, A., Kirsch, S., Henegariu, O., Hirschmann, P. \& Kiesewetter, F. et al. Human $Y$ chromosome azoospermia factors (AZF) mapped to different subregions in Yq11. Hum. Mol. Genet. 5, 933-943 (1996).

2 Giacco, D. L., Chianese, C., Sanchez-Curbelo, J., Bassas, L., Ruiz, P., Rajmil, O. et al. Clinical relevance of $Y$-linked CNV screening in male infertility: new insights based on the 8-year experience of a diagnostic genetic laboratory. Eur. J. Hum. Genet. 22, 754-761 (2014).
3 Repping, S., Skaletsky, H., Lange, J., Silber, S., Veen, F., Oates, R. D. et al. Recombination between palindromes $\mathrm{P} 5$ and $\mathrm{P} 1$ on the human $\mathrm{Y}$ chromosome causes massive deletions and spermatogenic failure. Am. J. Hum. Genet. 71, 906-922 (2002).

4 Kuroda-Kawaguchi, T., Skaletsky, H., Brown, L. G., Minx, P. J., Cordum, H. S., Waterston, $\mathrm{R}$. H. et al. The AZFc region of the $\mathrm{Y}$ chromosome features massive palindromes and uniform recurrent deletions in infertile men. Nat. Genet. 29, 279-286 (2001)

5 Lu, C., Jiang, J., Zhang, R., Wang, Y., Xu, M., Qin, Y. et al. Gene copy number alterations in the azoospermia-associated AZFc region and their effect on spermatogenic impairment. Mol. Hum. Reprod. 20, 836-843 (2014).

6 Shen, Y., Yan, Y., Liu, Y., Zhang, S., Yang, D., Zhang, P. et al. A significant effect of the TSPY1 copy number on spermatogenesis efficiency and the phenotypic expression of the gr/gr deletion. Hum. Mol. Genet. 22, 1679-1695 (2013).

7 Stahl, P. S., Mielnik, A. N., Barbieri, C. E., Schlegel, P. N. \& Paduch, D. A. Deletion or underexpression of they-chromosome genes CDY2 and HSFY is associated with maturation arrest in American men with nonobstructive azoospermia. Asian J. Androl. 14, 676-682 (2012).

8 Kleiman, S. E., Yogev, L., Hauser, R., Botchan, A., Maymon, B. B., Schreiber, L. et al. Members of the CDY family have different expression patterns: CDY1 transcripts have the best correlation with complete spermatogenesis. Hum. Genet. 113, 486-492 (2003).

9 Krausz, C., Hoefsloot, L., Simoni, M. \& Tuttelmann, F. EAA/EMQN best practice guidelines for molecular diagnosis of Y-chromosomal microdeletions: state-of-theart 2013. Andrology 2, 5-19 (2014)

10 Rosen, S. G., Marszalek, J. D., Irenze, K., Skaletsky, H., Brown, L. G. \& Oates, R. D. et al. AZFc deletions and spermatogenic failure: a population-based survey of 20,000 Y chromosomes. Am. J. Hum. Genet. 91, 890-896 (2012).

11 Carvalho, C. M. B., Zuccherato, L. W., Fujisawa, M., Shirakawa, T., Ribeiro-dos-Santos, A. K. C., Santos, S. E. B. et al. Study of AZFc partial deletion gr/gr in firtile and infertile Japanese males. J. Hum. Genet. 51, 794-799 (2006).

12 Repping, S., Skaletsky, H., Brown, L., Daalen, S. K. M., Korver, C. M., Pyntikova, T. et al. Polymorphism for a 1.6-Mb deletion of the human $\mathrm{Y}$ chromosome persists through balance between recurrent mutation and haploid selection. Nat. Genet. 35, 247-251 (2003)

13 Fernandes, S., Paracchini, S., Meyer, L. H., Floridia, G., Tyler-Smith, C. \& Vogt, P. H. A large AZFC deletion removes DAZ3/DAZ4 and nearby genes from men in $Y$ haplogroup N. Am. J. Hum. Genet. 74, 180-187 (2004).

14 Lu, C., Zhang, F., Yang, H., Xu, M., Du, G., Wu, W. et al. Additional genomic duplications in AZFc underlie the b2/b3 deletion-associated risk of spermatogenic impairment in Han Chinese population. Hum. Mol. Genet. 20, 4411-4421 (2011).

15 Krausz, C., Giachini, C., Xue, Y., O'Brya, M. K., Gromoll, J., Meyts, E. R. et al. Phenotypic variation within European carriers of the Y-chromosomal gr/gr deletion is independent of Y-chromosomal background. J. Med. Genet. 46, 21-31 (2009).

16 Lin, Y., Hsu, L. C., Kuo, P., Huang, W. J., Chiang, H., Yeh, S. et al. Partial duplication at AZFc on the $Y$ chromosome is a risk factor for impaired spermatogenesis in Han Chinese in Taiwan. Hum. Mutat. 28, 486-494 (2007).

17 Ye, J., Ma, L., Yang, L., Wang, J., Wang, Y., Guo, H. et al. Partial AZFc duplications not deletions are associated with male infertility in the $\mathrm{Yi}$ population of Yunnan Province, China. J. Zhejiang Univ. Sci. B 14, 807-815 (2013).

18 Giachini, C., Laface, I., Guarducci, E., Balercia, G., Forti, G. \& Krausz, C. Partial AZFc deletions and duplications: clinical correlates in the Italian population. Hum. Genet. 124, 399-410 (2008)

19 Stuppia, L., Antonucci, I., Palka, G. \& Gatta, V. Use of the MLPA assay in the molecular diagnosis of gene copy number alterations in human genetic diseases. Int. J. Mol. Sci. 13, 3245-3276 (2012).

20 Coughlin, C. R., Scharer, G. H. \& Shaikh, T. H. Clinical impact of copy number variation analysis using high-resolution microarray technologies: advantages, limitations and concerns. Genome Med. 4, 80 (2012).

21 Bunyan, D. J., Callaway, J. L. A. \& Laddach, N. Detection of partial deletions of Y-chromosome AZFc in infertile men using the multiplex Ligation-dependent Probe Amplification Assay. J. Reprod. Infertil. 13, 174-178 (2012).

22 Liu, X. H., Yan, L. Y., Lu, C. L., Li, R., Zhu, X. H. \& Jin, H. Y. ART do not increase the risk of Y-chromosome microdeletion in 19 candidate genes at AZF regions. Reprod. Fertil. Dev. 26, 778-786 (2013).

23 Kihaile, P. E., Yasui, A. \& Shuto, Y. Prospective assessment of Y-chromosome microdeletions and reproductive outcomes among infertile couples of Japanese and African origin. J. Exp. Clin. Assist. Reprod. 2, 9 (2005).

24 Carvalho, C. M. B., Zhang, F. \& Lupski, J. R. Structural variation of the human genome: mechanisms, assays, and role in male infertility. Syst. Biol. Reprod. Med. 57, 3-16 (2011).

25 Sun, C., Skaletsky, H., Birren, B., Devon, K., Tang, Z., Silber, S. et al. An azoospermic man with a de novo point mutation in the Y-chromosomal gene USP9Y. Nat. Genet. 23, 429-432 (1999).

26 Cram, D. S., Ma, K., Bhasin, S., Arias, J., Pandjaitan, M., Chu, B. et al. Y chromosome analysis of infertile men and their sons conceived through intracytoplasmic sperm injection: vertical transmission of deletions and rarity of de novo deletions. Fertil. Steril. 74, 909-915 (2000). 